\title{
Fuzzy FMEA Application Combined with Fuzzy Cognitive Maps to Manage the Risks of a Software Project
}

\author{
Barbaros Erbay \\ Coşkun Özkan
}

Industrial Engineering, Yıldız Technical University, Istanbul, Turkey

\begin{abstract}
The failure rate of an Information Technologies (IT) software project is pretty high because of their uncertain and risky structure. Managing well this kind of projects becomes important. Failure Mode and Effect Analysis (FMEA) is an extensive method that is used for identifying the importance level of risks in a project by using risk priority numbers (RPN). This method is based on experts' experience and cognitive skills at gathering data in order to make risk assessment. This situation causes inaccurate conclusions in the final risk ranking. Fuzzy logic is widely integrated into FMEA to handle these inaccuracies and inconsistencies in the literature while making assessment and calling Fuzzy FMEA method that we proposed. In this study, we explored another uncovered weaknesses of the proposed method. FMEA and Fuzzy FMEA do not consider the relationships among the risks of a project. To overcome this disadvantage, we proposed to integrate the idea of cognitive maps into these two methods (FMEA w/FCMs and Fuzzy FMEA w/FCMs). Finally, we got a comprehensive risk assessment methodology by considering the relationships among the risks under ambiguous circumstances.
\end{abstract}

Keywords: FMEA, Fuzzy logic, Fuzzy cognitive maps, Risk analysis.

\section{Introduction}

Information technologies have an important role in business life. To be able to compete with other companies, a company needs to have a successful information technology (IT). A successful IT construction can come true after a successful IT project. IT projects are high risky, complicated, expensive and they have uncertainty conditions. Because of these reasons IT projects have a high rate about being unsuccessful.

Companies need to manage well the risks of their IT projects under environmental conditions with high uncertainty, discrete small and incomplete data sets and lack of knowledge. There are some methods to manage the risks in literature. One of them is Failure Mode and Effect Analysis (FMEA). FMEA is an effective and highly used method to make risk assessment. FMEA uses experts' views to make risk prioritization by finding Occurrence value (O), Severity value (S) and Not Detection value (D). FMEA calculates the Risk Priority Number (RPN) values by multiplying $O, S$ and $D$ values and finally sorts the RPN values by descending. This helps analysts to manage the risks of IT project. They easily realize the risks which they need to handle at first. But FMEA have some weaknesses while making risk assessment.

Fuzzy Logic is a method of reasoning that resembles human reasoning. Fuzzy logic produces acceptable but definite output in response to incomplete, ambiguous or inaccurate input. To overcome weaknesses of FMEA, there are lots of successful applications which contain fuzzy logic and FMEA together in literature. These methods aim to manage the risks of IT projects in spite of all deficiencies and uncertainty. On the other hand this method doesn't consider the relationships among the risks as a weakness. 
Fuzzy Cognitive Maps (FCMs) is a technique that is used for modeling complicated systems and representing the cause and effect relationships among the components of complicated systems. In that study FCMs method is used in order to overcome the weakness of Fuzzy FMEA method. This final method calls as Fuzzy FMEA with FCMs. This new method aims to make risk management to consider the relationships among the risks in an IT project.

\section{Literature Review and General Information}

\subsection{Failure Mode and Effect Analysis}

FMEA is widely used in manufacturing industries in various phases of the product life cycle and is now increasingly finding use in the service industry(1). To help reliability-related problems, FMEA has been widely used in various manufacturing areas (2). In recent years FMEA has increased its scope and it is applied in service sector (3). In service sector, FMEA was offered providing the generic guidelines required applying to the service setting together with system FMEA, design FMEA and process FMEA (4). FMEA method was applied to IT Projects in service sector and they took interpretable results from it.

Moreover, FMEA is integrated to other methods in the literature. Braglia (2000) (5) extended FMEA method which is called Multi Attribute Failure Mode Analysis. Author has embedded FMEA to Analytic Hierarchy Process (AHP) in order to define importance levels of failure modes. Pillay and Wang (2003) (6) suggested a new approach and this approach covers Fuzzy logic and grey theory with FMEA. To weight the risk factor values, Chang (2009) (7) suggested the ordered geometric averaging method (OWGA) and decision making trial and evaluation laboratory method (DEMATEL) to make prioritization the failure modes in FMEA.

FMEA is a reliability tool that is used for defining potential failures before they occur to minimize the risks' effects (8) (9). The purpose of evaluation in FMEA method is to define the risks numerically which will occur and to prioritize them. In that stage the criticality level of each risk is defined, independently. In traditional FMEA, a risk priority number (RPN) is calculated to evaluate the risk level of a component/process (1). After RPN values are calculated, the results are sorted in by descending order. Since the higher value of RPN means that the associated risk is more critical, the resulting order helps analyst to investigate the solutions for preparedness and to determine the prevention and/or mitigation plans before risk occurrence. The RPN is obtained by finding the multiplication of three factors, as given in $R P N=O * S * D$

$$
\text { (2.1): }
$$

Representing this mathematically will give:

$R P N=O * S * D$

where $O$ denotes the probability/occurrence of the failure, $S$ denotes the severity of the failure and $D$ denotes the probability of not detecting the failure. The process of FMEA is given in (Figure 2.1 FMEA Method).

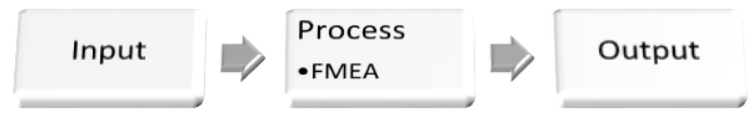

Figure 2.1 FMEA Method

\subsection{Fuzzy FMEA}

There are important applications have been made in FMEA literature to overcome the shortcomings of the traditional RPN (10). Fuzzy FMEA logic uses experts' view who describe the risk factors $O, S$ and $D$ by using the fuzzy linguistic terms. To evaluate three risk factors $O, S$ and $D$ the linguistic variables were used. Bowles and Pelaez described a fuzzy logic based approach for prioritizing failures in FMEA which uses fuzzy linguistic terms to describe $O, S$ and $D$ and the risks of failures (11). According to expert knowledge, fuzzy if-then rules were obtained and expertise provided finding the relationships between a risk and its $O, S$ and $D$ values for every risk. Fuzzification process was run for crisp ratings for $O, S$ and $D$ to match the premise of each possible if-then rule. All the rules that have any truth in their premises were fired to contribute to fuzzy conclusion. The defuzzification process was finally applied to get the fuzzy conclusion the weighted mean of maximum method as the ranking value of risk priority (1). 
Pillay and Wang (6) proposed a fuzzy rule base approach to avoid the use of traditional RPN. They tried to set up the membership functions of the three risk factors $\mathrm{O}, \mathrm{S}$ and $\mathrm{D}$. Membership functions have been developed and FMEA is applied in its traditional way with the use of brainstorming techniques. Each failure mode is assigned a linguistic term for each of the three risk factors. The three linguistic terms are integrated using the fuzzy rule base generated to produce a linguistic term representing the priority for attention. This linguistic term represents the risk ranking of the failure mode.

Fuzzy logic is a form of multi-valued that is obtained from fuzzy set theory to overcome reasoning that is approximate rather than precise (1). The fuzzy logic variable may have a membership value not only 0 or 1 but also a value inclusively between 0 and 1 (1). In fuzzy logic the degree of truth of a statement can range between 0 and 1 and is not constrained to the two truth values \{true (1), false (0)\} as in classic propositional logic (1). Approximate reasoning which is a made of reasoning that is not exact or very inexact is a basis provided by the fuzzy logic (1). The fuzzy logic proposes a more down to earth framework for reasoning than the traditional two-valued logic.

The name of fuzzy logic emerged by Lotfi Zadeh (12) as an outcome of the development of the theory of fuzzy sets. In 1965 , Zadeh proposed fuzzy set theory (12), and later established fuzzy logic based on fuzzy sets. The process of fuzzy logic is given in (Figure 2.2 The methodology of Fuzzy FMEA) (1).

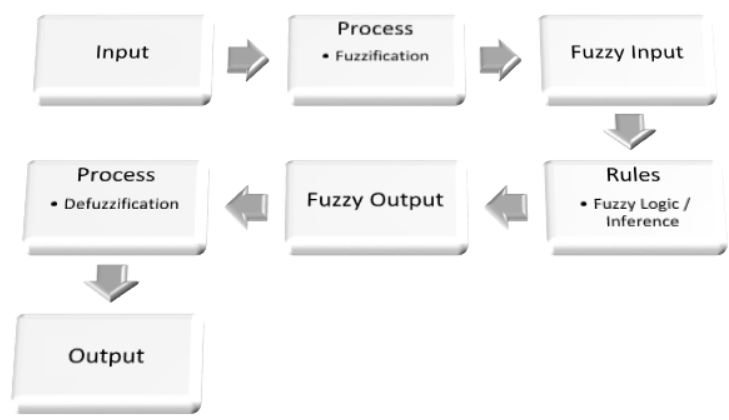

Figure 2.2 The methodology of Fuzzy FMEA

In the proposed approach, a fuzzy rule base is used to rank the potential causes identified within the FMEA, which would have identical RPN values but different risk implications. The approach then extends the analysis to include weighting factors for $O, S$ and $D$ using defuzzified linguistic terms.

Algorithm of fuzzy logic is as follows:

1) Calculate average $O, S, D$ values for every risk $(\bar{O}, \bar{S}, \bar{D})$.

2) Find the membership functions and function levels for every input variable of risks.

3) Get the results according to membership function that is used.

4) Use Mamdani min/max method of inference mechanism and find the function levels and the minimum input value among $\bar{O}, \bar{S}, \bar{D}$ values for every risk.

5) Find the function levels for output function by using output rules table.

6) Defuzzify the results by using center of gravity method.

\subsection{Fuzzy Cognitive Maps (FCMs)}

The origin of FCMs is the concept of CMs which is first proposed by Tolman (13). In order to represent the cause and effect relationships among the elements of a given environment in political and social sciences CMs has been applied (13) (14). Then, Axelrod claimed that $\mathrm{CM}$ with causality value + and - is adequate for simulating human cognition and following this decision makers don't tend to prefer more complicated set of relationships to solve problems(14).

Kosko (15) proposed the Fuzzy Cognitive Maps (FCMs) technique in order to evolve a CMs model because of two important conditions. 
Fuzzy logic can make casual relationships between nodes have different intensities. An uncertain value is more preferred rather than an exact value. Because of this reason, Kosko (16) proposed the Fuzzy Cognitive Maps (FCMs) technique in order to evolve a CMs model. A fuzzy number in that model can have a value between 0 and 1 or -1 and 1 , including both (17). Each numerical value in the interval represents the grade of membership to a fuzzy set, where 0 is the nonmembership and 1 the full membership (18). In addition to them, in FCMs, there is an initial vector of nodes which contains initial values at the instant 0 and the model simulate this vector at the instant $t$ until it reaches a stable vector. So this technique can forecast the future behaviors of a system which is working on. In addition, FCMs provide excellent mechanisms to develop forecasting exercises. Specifically, this technique enables us to develop what-if analysis, supporting the critical decision-making (19).

The nodes show dynamic variables in a dynamic system. The edges show directions and intensity of casual relationship among the variables. Each cause is assessed by its intensity $w_{i j}$, where $i$ is the pre-synaptic (causal) node and $j$ the postsynaptic (effect) node. The $w_{i j}$ values are represented in the $n x n$ ( $n$ is the number of nodes) matrix called adjacency $\mathrm{m}$

$\begin{aligned} & \mathrm{a} \\ & \mathrm{r} \\ & \mathrm{r}\end{aligned}=\left(\begin{array}{ccc}\ldots & \ldots & \ldots \\ \ldots & \boldsymbol{w}_{i j} & \ldots \\ \ldots & \ldots & \ldots\end{array}\right)$

There are three possible types of causal relationships between nodes (17):

） $\quad w_{i j}>0$ : Positive causality between nodes $x_{i}$ and $x_{j}$.

- $\quad w_{i j}<0$ : Negative causality between nodes $x_{i}$ and $x_{j}$.

$\mathrm{S}$

- $\quad w_{i j}=0$ : No causal relationship exists between nodes $x_{i}$ and $x_{j}$.

A is possible to develop forecasting exercises, especially what-if analysis in FCMs. For this purpose, what-if scenarios at the instant $t=0$ are defined. In this way, the values of all nodes of FCM are entered in a $1 \times n$ initial state vector $C^{t}$, see $C t=\left(C_{1}^{t}, C_{2}^{t}, \ldots, C_{i}^{t}, \ldots, C_{n}^{t}\right)$

(2.3). The value of each node in the input vector can be 1 (element is

activated) or 0 (element is not activated) (17).

$\mathrm{E}^{t}=\left(C_{1}^{t}, C_{2}^{t}, \ldots, C_{i}^{t}, \ldots, C_{n}^{t}\right)$

where $C^{t}$ is the initial vector state (at the instant $t$ ), and $C_{i}^{t}$ is the initial value of the $i$ node (at the instant $t$ ) (17).

$\stackrel{S}{R}$

Q

6

f

Activation functions such as the sigmoid, hyperbolic tangent, step and threshold linear can be used in the FCM inference frocess (20), (21), (22).

$\sum_{j}^{t+1}=f\left(\sum_{\substack{i=1 \\ i \neq j}} C_{i}^{t} * w_{i j}\right)$

ghere $f(x)$ is the activation function, $C_{j}^{t+1}$ the value of the post-synaptic (effect) node $j$ at the instant $t+1, C_{i}^{t}$ the value of the pre-synaptic (causal) node $i$ at the instant $t$, and $w_{i j}$ indicate the intensity of the relationships between the preyynaptic (causal) node $i$ and the post-synaptic (effect) node $j(17)$.

The nonlinear function $\mathrm{f}$ allows the activation to take an allowed value. In this study, we used sigmoid function.

\section{b Integrated Methodology for Risk Assessment: Fuzzy FMEA Integrated with Fuzzy Cognitive Maps}

EMEA and Fuzzy FMEA processes are used for defining the importance of risks of projects but all these processes have a weakness. They don't consider the relationships among risks of projects. In order to overcome this weakness we used Fuzzy Cognitive Maps by extending of these processes. For this extension we aimed to reach an extension coefficient to define a new Severity (S) value when the risks of a project affect each other by using Fuzzy Cognitive Maps. By this way the relationships among risks are considered to overcome the weakness of FMEA and Fuzzy FMEA. All these processes gre applied after this extension.

$\mathrm{R}$ 
The last $C_{j}^{t}$ value shows impact of nodej. At the end of FCM process every node reach an affected value. According to activation function that is chosen by practitioner, there is a sub limit of nodes. It means if the beginning value of node $j$ equals to zero, the result will be equal to sub limit. According to these values that are obtained at the end of FCM process, the coefficient value is calculated for every node. It means every risk will have a coefficient value and severity value of every risk will be calculated by these coefficient values.

$\lambda_{j}=\delta_{j}-\varphi$

where $\varphi$ is the sub limit of nodes. $\delta_{j}$ is the last $C^{t}$ value of node $j . \lambda_{j}$ is the coefficient value of the risk $j$. A new Severity v

$\vec{u}_{j}^{\prime}=\left\{\begin{array}{l}S_{j} *\left(1+\lambda_{j}\right), S_{j} *\left(1+\lambda_{j}\right)<10 \\ 10 \quad, S_{j} *\left(1+\lambda_{j}\right) \geq 10\end{array}\right.$

where $S_{j}^{\prime}$ is the new severity value that will be used for calculating the new importance levels by FMEA, and Fuzzy FMEA.

$\mathrm{F}$

㟶 $P N_{j}^{\prime}=O_{j} * S_{j}^{\prime} * D_{j}$

The process of FMEA is given in (Figure 2.1 FMEA Method). After FMEA process is expanded by Fuzzy Cognitive Maps the new FMEA process is given (Figure 3.1 FMEA Process by FCM).

$x$

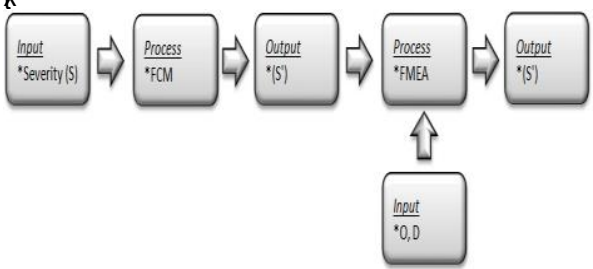

4

Pigure 3.1 FMEA Process by FCM

The process of Fuzzy FMEA is given in (Figure 2.2). After Fuzzy FMEA process is expanded by Fuzzy Cognitive Maps the hew Fuzzy FMEA process is given (Figure 3.2).

a 


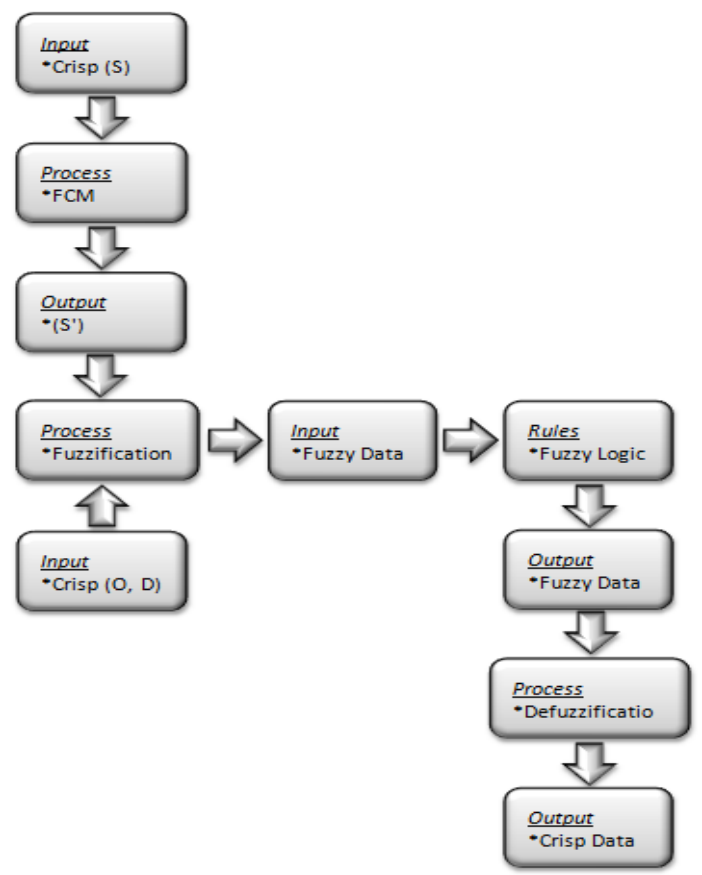

Figure 3.2 Fuzzy FMEA Process by FCM

\section{Case Study}

\subsection{Data Collection}

IT software projects have risky, complex and hard-to-understand structures for managing by project managers. That's why risk management plays an important role to achieve projects' goals successfully.

We will investigate the risks of a real IT software project. The top management of a company needs a new software application to assign tasks, to follow users' tasks, to watch the current situation and to have reports about these tasks. They have decided to develop an in-house project which satisfies their requirements by IT department. So all these processes have risks and these risks need to be managed. At the end of this investigation we will put them in order according to their importance and then we will make suggestions to managers to lead this project. In this investigation FMEA and Fuzzy FMEA were used as known methods. Risk prioritizations of these methods were compared and interpreted. In addition to this, to consider the relations among the risks we integrated Fuzzy Cognitive Maps to every method and then we compared all results to observe the changing risks' priorities.

For this project we specified 23 risks as follows:

Table 4.1 Risks of IT Project

\begin{tabular}{ll}
\hline Risk Code & Risk \\
\hline R1 & Conflicts between organization and consultants/ vendor \\
R2 & High rate of system customization \\
R3 & Data management issues \\
R4 & Inadequate education and training \\
R5 & Inadequate user involvement \\
R6 & Ineffective communications system \\
R7 & Internal conflicts between departments \\
R8 & Inadequate change management \\
R9 & Lack of performance measurement system \\
R10 & Misfit between organization culture and ERP system \\
R11 & Misfit between organization structure and ERP system \\
\hline
\end{tabular}




\begin{tabular}{ll}
\hline R12 & Misfits between the IT and business strategies \\
R13 & Environmental pressures \\
R14 & Poor business process reengineering \\
R15 & Poor consultant \\
R16 & Poor project management \\
R17 & Poor risk management \\
R18 & Poor top management support \\
R19 & IT Technical issues \\
R20 & Language barriers \\
R21 & Poor project team \\
R22 & Poor knowledge transfer \\
R23 & Poor quality of testing \\
\hline
\end{tabular}

These risks are defined according to character of the case in point. So for other projects, the risks need to be characterized according to conditions of the case or problem.

\subsection{Application of New Method: Fuzzy FMEA Integrated with FCMs}

To make prioritization we used a new method called Fuzzy FMEA and we also surveyed its stability the whether it is applicable or not. The simulation results showed us that it is a suitable method to assessment risks for projects under conditions with high uncertainty, under discrete small and incomplete data sets. After all this method still have a weakness that actually comes from nature of FMEA. This weakness is ignoring the relationships among the risks. FMEA and Fuzzy logic in that study are not enough to overcome that. As stated above in order to consider the relationships among the risks we used Fuzzy Cognitive maps.

In order to measure effects of FCMs on the other methods, we firstly applied it to FMEA and compared the results of two methods in themselves. Then secondly we applied it to Fuzzy FMEA and again compared the results of two methods in themselves. Finally Fuzzy FMEA integrated with Fuzzy Cognitive Maps is the goal that we want to reach. Comparisons in themselves of every couple methods also show that the integration process is a practicable process.

\subsubsection{Fuzzy Cognitive Maps}

This approach consists in adding the FCM drawing by each expert. A group of experts was carefully selected to participate in our study. Each expert individually designed his/her own FCM model, which represent his/her knowledge in IT projects. They thus pointed out which risks had threatened their projects' risks. The experts also drew the interactions that exist between IT project risks nodes. That is, they specified the type and intensity of the casual relationships existing among nodes. Experts can indicate the causal connections using linguistic variables or real numbers. Those participating in the present study expressed all relations with a numerical value in a range of $[-1,1]$. We thus achieved one adjacency matrix for each expert.

The Augmented FCM method finishes by adding the adjacency matrices of each one of them. This depends on if there are or are not common nodes. If there are not common risks, adjacency matrices will be solely added up. Otherwise, if there are common nodes, then the elements $w_{i j}^{A U G}$ in the augmented matrix $\left(A^{A U G}\right)$ are computed according to the following wijAUG $=\frac{\sum_{i=1}^{m} w_{i j}^{k}}{m}$

$w_{i j}^{A U G}=\frac{\sum_{i=1}^{m} w_{i j}^{k}}{m}$

where $m$ is the number of FCMs added, one per expert, $k$ is the identifier for each FCM, and $i$ and $j$ are identifiers of the connections.

We computed the elements for the $A^{A U G}$ using (see Appendix A) because the experts' FCM had common nodes.

You can reach all experts' views by this link:

https://drive.google.com/file/d/OB-OGfN4no_-TVmw4TTNmMUt0cig/view?usp=sharing

Subsequently, we compared the FCM obtained with respect to the research conceptual framework to guarantee the logical validation. For the partial graphical representation of the model, see Appendix $B$. 
In this way, we applied the hyperbolic tangent function Error! Reference source not found.), with a function slope $(\lambda)$ equal to 1 in the FCM simulations. The value of the FCM nodes is located within the range $[0,1]$ because we chose the risks according to positive causality or no causality. There is no negative causality among the nodes. In addition, this usually requires a lower number of interactions to reach a stable scenario in comparison to other activation functions.

In

Finally,

Fuzzy

These

R13

Interaction

Interaction

4.2.4 Getting New Severity Values

Interaction

\section{To}

Firstly we have indicated a non-interactivity limit of nodes. This is 0.500 and it is a feature of the sigmoid function. Then we calculated the coefficient values of risks by using the non-interactivity limit $\lambda j=\delta_{j}-\varphi$

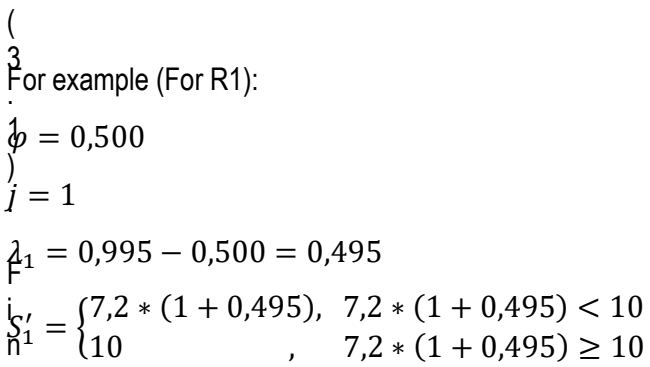

in that example, the new Severity value would be more than 10 but in FMEA O, S, D values' range is between 1 and 10 . That's why we set 10 the value when the value is more than 10 .

$\$_{1}^{\prime}=10(10,764 \geq 10)$

$R P N_{1}^{\prime}=3 * 10 * 6=180$

Åccording to this information, for all risks, the new $S$ values $\left(S^{\prime}\right)$ would be like in Appendix $F$.

$\boldsymbol{m}$ addition, if the value of a node was under 0,500 at the end all simulations, its coefficient value would be negative and it brould make new severity value of the node less than old severity value.

\subsubsection{FMEA Integrated with Fuzzy Cognitive Maps}

The evaluation of the failure modes is carried out by scoring the respective risk factors of occurrence (O), severity (S), and hot detection (D). For this purpose, usually 10-level scales are being used. While scoring the risk factors a variety of statistical techniques or expert opinion is referred to. In this study, all the risk factors were based on expert opinion.

d

In this project we have 5 experts and we asked to them O, S, D values for every risks and finally we calculated the arithmetic mean of their opinions to use them in FMEA application.

When we applied FMEA to the risks, the results are as follows in Table 4.2 as Old RPN and Old Prioritization. e

According to FMEA results, while project managers consider the risks, they need to be careful R8, R2, R23, R19 and R14. If we assume that risks which their RPN value are above 300 are important, we could say that these risks has critical importance level according to FMEA method.

$\mathrm{R} 17$ - R22 and R3 - R7 risk groups have different risk factor values in themselves but their RPN values are same. It means they need to be evaluated in same level despite the fact that they have different values. 
Table 4.2 Results of FMEA Integrated with FCM (Comparison)

\begin{tabular}{lllllll}
\hline Risks & Old RPN & $\begin{array}{l}\text { Old } \\
\text { Prio } \\
\text { ritiza } \\
\text { tion }\end{array}$ & New RPN & $\begin{array}{l}\text { Effect } \\
\text { RPN }\end{array}$ & $\begin{array}{l}\text { on } \\
\text { New } \\
\text { Prio } \\
\text { ritiza } \\
\text { tion }\end{array}$ \\
\hline R8 & 662,888 & 1 & 770,800 & 107,912 & 1 \\
R2 & 512,992 & 2 & 557,600 & 44,608 & 2 \\
R23 & 392,496 & 3 & 479,834 & 87,338 & 3 \\
R22 & 276,08 & 7 & 394,400 & 118,320 & 4 \\
R19 & 337,92 & 4 & 384,000 & 46,080 & 5 \\
R17 & 276,08 & 6 & 357,539 & 81,459 & 6 \\
R14 & 313,2 & 5 & 348,000 & 34,800 & 7 \\
R21 & 266,112 & 8 & 316,800 & 50,688 & 8 \\
R16 & 202,176 & 10 & 280,800 & 78,624 & 9 \\
R5 & 167,04 & 12 & 219,188 & 52,148 & 10 \\
R13 & 209,088 & 9 & 209,088 & 0,000 & 11 \\
R7 & 126,72 & 14 & 189,819 & 63,099 & 12 \\
R9 & 174,064 & 11 & 180,824 & 6,760 & 13 \\
R1 & 120,96 & 15 & 168,000 & 47,040 & 14 \\
R6 & 119,784 & 16 & 163,669 & 43,885 & 15 \\
R3 & 126,72 & 13 & 144,000 & 17,280 & 16 \\
R11 & 101,376 & 17 & 139,539 & 38,163 & 17 \\
R18 & 90,72 & 18 & 129,600 & 38,880 & 18 \\
R12 & 73,728 & 19 & 99,847 & 26,119 & 19 \\
R10 & 53,76 & 21 & 79,910 & 26,150 & 20 \\
R15 & 63,36 & 20 & 74,574 & 11,214 & 21 \\
R4 & 28,16 & 22 & 39,824 & 11,664 & 22 \\
R20 & 13,552 & 23 & 15,496 & 1,944 & 23 \\
\hline R & 23 & & \\
\hline
\end{tabular}

While making prioritization we considered the old prioritization. Otherwise the risks that their RPN values are equal to each other would have randomly been ordered in themselves.

According to results, there are two important effects above 100 (R8: 107,912 and R22:118,320) by means of this integration of FCM haven changed prioritization of R22. It made its prioritization from 7 to 4 . It means integration of FCM have made it more important risk. There is one more risk like R22 but this time Integration of FCM have made it more unimportant risk by changing its priority from 13 to 16 (R3). These two risks have the largest changes $(7-4=3=16-13)$ according to other risks' changes. That means FCM didn't make a dramatic change.

In FMEA, R17 and R22 have same RPN values and their prioritizations are respectively 6 and 7 . After integration of FCM R17 kept its place same (6) but R22 became more important risk (4) as mentioned above. There is a similar situation with a little difference for R3 and R7. In FMEA their RPN values are same and their prioritizations are respectively 13 and 14 . After FCM while R7 increased its priority from 14 to 12, R3 has lost its importance a little bit and became from 13 to 16. This shows that when we considered the relationship among the risks this application could change their prioritizations. So we can conclude that integration of FCM can affect the risks in three ways:

- $\quad \mathrm{FCM}$ can increase risks' importance levels: $R 22(7 \rightarrow 4), R 16(10 \rightarrow 9), R 5(12 \rightarrow 10), R 7(14 \rightarrow 12), R 1(15 \rightarrow 14), R 6$ $(16 \rightarrow 15), R 10(21 \rightarrow 20)$

- $\mathrm{FCM}$ can decrease risks' importance levels: $R 19(4 \rightarrow 5), R 14(5 \rightarrow 7), R 13(9 \rightarrow 11), R 9(11 \rightarrow 13), R 3(13 \rightarrow 16), R 15$ $(20 \rightarrow 21)$

- $\quad$ FCM can keep same risks' importance levels: $R 8(1 \rightarrow 1), R 2(2 \rightarrow 2), R 23(3 \rightarrow 3), R 17(6 \rightarrow 6), R 21(8 \rightarrow 8), R 11$ $(17 \rightarrow 17), R 18(18 \rightarrow 18), R 12(19 \rightarrow 19), R 4(22 \rightarrow 22), R 20(23 \rightarrow 23)$

\subsubsection{Fuzzy FMEA Integrated With Fuzzy Cognitive Maps}

A model was established for the FMEA technique having 3 inputs and 1 output variable. The RPN values were calculated by combining the associated 3 input factors. For the input variables of occurrence, severity and not detection a 5 -level; and for the output variable RPN a 10-level triangular membership functions.

For input values, the 10-level scale is stated 5 regions as triangular membership functions. Input variables' membership functions would be as below (Almost None, Low, Medium, High, Very High): 
Table 4.3 Membership Functions of Input Variables

\begin{tabular}{lll}
\hline Membership & Function & Limits \\
\hline Almost $N$. & $\mu(x)=(2-x) / 2$ & $0,00 \leq x \leq 2,00$ \\
Low 1 & $\mu(x)=(x-1) /(3 / 2)$ & $1,00 \leq x \leq 2,50$ \\
Low 2 & $\mu(x)=(4-x) /(3 / 2)$ & $2,50 \leq x \leq 4,00$ \\
Medium 1 & $\mu(x)=(x-3) / 2$ & $3,00 \leq x \leq 5,00$ \\
Medium 2 & $\mu(x)=(7-x) / 2$ & $5,00 \leq x \leq 7,00$ \\
High 1 & $\mu(x)=(x-6) /(3 / 2)$ & $6,00 \leq x \leq 7,50$ \\
High 2 & $\mu(x)=(9-x) /(3 / 2)$ & $7,50 \leq x \leq 9,00$ \\
Very High & $\mu(x)=(x-8) / 2$ & $8,00 \leq x \leq 10,00$
\end{tabular}

For output values, the 10-level scale is stated 10 regions as triangular membership functions. Output variables' membership functions would be as below (None, Very Low, Low, High Low, Low Medium, Medium, High Medium, Low High, High, Very High):

Table 4.4 Membership Functions of Output Variables

\begin{tabular}{lll}
\hline Membership & Function & Limits \\
\hline None & $\mu(x)=(2-x) / 2$ & $0,00 \leq x \leq 2,00$ \\
Very Low 1 & $\mu(x)=x-1$ & $1,00 \leq x \leq 2,00$ \\
Very Low 2 & $\mu(x)=3-x$ & $2,00 \leq x \leq 3,00$ \\
Low 1 & $\mu(x)=x-2$ & $2,00 \leq x \leq 3,00$ \\
Low 2 & $\mu(x)=4-x$ & $3,00 \leq x \leq 4,00$ \\
High Low 1 & $\mu(x)=x-3$ & $3,00 \leq x \leq 4,00$ \\
High Low 2 & $\mu(x)=5-x$ & $4,00 \leq x \leq 5,00$ \\
Low Medium 1 & $\mu(x)=x-4$ & $4,00 \leq x \leq 5,00$ \\
Low Medium 2 & $\mu(x)=6-x$ & $5,00 \leq x \leq 6,00$ \\
Medium 1 & $\mu(x)=x-5$ & $5,00 \leq x \leq 6,00$ \\
Medium 2 & $\mu(x)=7-x$ & $6,00 \leq x \leq 7,00$ \\
High Medium 1 & $\mu(x)=x-6$ & $6,00 \leq x \leq 7,00$ \\
High Medium 2 & $\mu(x)=8-x$ & $7,00 \leq x \leq 8,00$ \\
Low High 1 & $\mu(x)=x-7$ & $7,00 \leq x \leq 8,00$ \\
Low High 2 & $\mu(x)=9-x$ & $8,00 \leq x \leq 9,00$ \\
High 1 & $\mu(x)=x-8$ & $8,00 \leq x \leq 9,00$ \\
High 2 & $\mu(x)=10-x$ & $9,00 \leq x \leq 10,00$ \\
Very High & $\mu(x)=x-9$ & $9,00 \leq x \leq 10,00$ \\
\hline How to Get Output $R u S$ & \\
\hline
\end{tabular}

\section{How to Get Output Rules}

To get output values, we have developed a new logic. In that way, every output values will have a mathematical calculation and same logic with the others.

Firstly, we have divided low, medium and high functions of input variables into two-side functions and then we have defined mathematical notations of input variables' membership functions (as it is seen Table 4.3).

After we got the functions, we have calculated CoG values (center of gravity) of every input function.

\begin{tabular}{|l|c|}
\hline Almost None (AN) & $\operatorname{CoG} G_{(A N)}=0,67$ \\
\hline Low (L) & $\operatorname{CoG}(L)=2,50$ \\
\hline Medium (M) & $\operatorname{CoG}(M)=5,00$ \\
\hline High (H) & $\operatorname{Co} G_{(H)}=7,50$ \\
\hline Very High (VH) & $\operatorname{CoG}(H)=7,50$ \\
\hline
\end{tabular}


After input variables were processed we have divided out of none and very high functions of output variables into two-side functions and then we have defined mathematical notations of output variables' membership functions (as it is seen Table 4.4).

We have calculated CoG values (center of gravity) of every output function to define ranges of output functions.

\begin{tabular}{lll}
\hline Output Variables & COG & Ranges \\
\hline None & 0,67 & $0,00-1,33$ \\
Very Low & 2,00 & $1,33-2,67$ \\
Low & 3,00 & $2,67-3,33$ \\
High Low & 4,00 & $3,33-4,67$ \\
Low Medium & 5,00 & $4,67-5,33$ \\
Medium & 6,00 & $5,33-6,67$ \\
High Medium & 7,00 & $6,67-7,33$ \\
Low High & 8,00 & $7,33-8,67$ \\
High & 9,00 & $8,67-9,33$ \\
Very High & 9,67 & $9,33-10,00$ \\
\hline
\end{tabular}

After we calculated CoGs and ranges of functions we have taken averages of every combination and then according to average values we have found fuzzy output function of every combination. Here is the Output rules table:

https://drive.google.com/file/d/12vNv3OQi4qmRefY2EmjNUcuGGpuvbjRA/view?usp=sharing

Results of Fuzzy FMEA

As to the types of failure, the fuzzy RPN values provided in the model are given in a descending order in (Table 5.5 Results of Fuzzy FMEA) in comparison with the RPN values of classical FMEA. The failure types containing the same RPN values were arranged according to the values of occurrence, severity and not detection (priority queues).

According to results the first 3 risks' prioritizations and the last 6 risks' prioritizations didn't change but there are some prioritization variations for other risks but in general results show two methods have similar risk prioritizations. In Fuzzy FMEA, priorities of R17 $(6 \rightarrow 4)$, R22 $(7 \rightarrow 5)$ and R21 $(8 \rightarrow 6)$ has increased two steps according to FMEA. For R9, R19 and R14 we can say that they have the biggest changes in comparison of two methods. While R9 has increased its priority from 11 to $7, \mathrm{R} 19$ and R14 have decreased their priorities four steps $(R 19: 4 \rightarrow 8, R 14: 5 \rightarrow 9)$. In addition to that R5 has decreased its priority three steps. There is just one-step change for priorities of R13, R16, R3, R7 and R1. In general we can say there is 0 an important variation.

As an example, R3 and R7 have same RPN values $(126,72)$ while they have different risk factor values $\left(R 3: 2,00^{*} 8,80^{*} 7,20=126,72, R 7: 6,60^{*} 4,80^{*} 4,00=126,72\right)$. According to FMEA, they need to be evaluated at same risk level and Fuzzy FMEA has set new risk levels for each risk but they still follow each other.

As a consequence Fuzzy FMEA can keep a risk's priority stable or can decrease/increase it.

In this study we have to be careful while prioritizing the risks. For example, difference between FMEA RPN values of R19 $(337,92)$ and $R 1(120,96)$ is 216,96 even though they have same Fuzzy RPN values $(6,00)$. For this study we can say it is pretty much difference. If we prioritized the risk by looking only Fuzzy RPN values, it would be a random prioritizing and consequently their priorities would take any number between 2 and 8 . In response to this while prioritizing the risks we consider FMEA priorities of the risks. We firstly sort the risks according to Fuzzy RPN values and then we sort them again according to their FMEA priorities.

We have the new severity ( $S^{\prime}$ ) values from previous section to search how integrating FCM affects the Fuzzy FMEA results. This is just to apply Fuzzy FMEA method with new severity values. Membership functions, rule base for fuzzy output and methods in Fuzzy are same (Table 4.4 Membership Functions of Output Variables. 
According to results, R8, R2, R23, R21, R16, R1, R11, R18, R12, R15 and R20 have still same Fuzzy RPN values and prioritizations. Integration of FCM to Fuzzy FMEA increased the importance levels of the risks: R22, R7, R5, R6 and R4 but however it decreased the importance levels of the risks: R17, R9, R19, R14, R13, R3, R10.

To put in a nutshell, the risk R7 has the largest dramatically variation. Its priority became from 13 to 7 . That means it became much more important risk than before. Also its Fuzzy RPN values increased to 7,000 from 6,000. However the risk R3 has same Fuzzy RPN values in two methods but its priority has fallen back to 16 from 12 . These two risks have same RPN values and same Fuzzy RPN values. But when considered the relationships among the risks they are taking different positions and behaviors.

However we can conclude that there is no dramatically change as well as FMEA integrated with FCM.

There are some priority changes though their RPN values (Fuzzy RPN and Re-Fuzzy RPN) are equal in themselves. For example R9 has same RPN values $(7,000)$ but while its priority number is 7 in Fuzzy FMEA, its priority number is 8 in Fuzzy FMEA integrated with FCM. While making prioritization in Fuzzy FMEA we considered FMEA prioritization as mentioned above. This is also valid for Fuzzy FMEA with FCM. While making its prioritization we also considered prioritization of FMEA with FCM. That's why this risk's priority is different.

\section{Conclusion}

To sum up all processes, in the literature there are many methods to make risk assessment. In this study we investigated two of them: FMEA and Fuzzy FMEA. FMEA is a technique to make prioritization by descending RPN values which are taken by multiplying $O, S$ and $D$ values of risks.

To overcome the shortcomings of the traditional RPN, fuzzy logic is widely used in the literature. Fuzzy FMEA logic uses experts' view who describe the risk factors $O, S$ and $D$ by using the fuzzy linguistic terms. In this study, we applied these two methods (FMEA and Fuzzy FMEA) to the real IT case and compared the results. Results showed us that there are some changes (not so dramatic) and the analysts can have a better and deeper method while making risk assessment.

In spite of this, these two methods have a weakness. They don't consider the relations among the risks. That's why we have decided to integrate Fuzzy Cognitive Maps to overcome this weakness of these methods. FCMs can make casual relationships between nodes have different intensities. The nodes show dynamic variables in a dynamic system. The edges show directions and intensity of casual relationship among the variables. In this way, we can observe when a risk happened, how affects the other risks.

After we applied these methods to the case by integrating FCM to each of them and compared the results. Results showed us that there are much more changes according to previous two methods but these changes are not so dramatic in general. That also means the new method can be used by practitioners to make risk assessment.

Table 5.5 Results of Fuzzy FMEA

\begin{tabular}{llllllll}
\hline Risks & 0 & S & D & RPN & $\begin{array}{l}\text { FMEA } \\
\text { Prioritization }\end{array}$ & Fuzzy RPN & $\begin{array}{l}\text { Fuzzy } \\
\text { Prioritization }\end{array}$ \\
\hline R8 & 8,20 & 8,60 & 9,40 & 662,89 & 1 & 9,000 & 1 \\
R2 & 6,80 & 9,20 & 8,20 & 512,99 & 2 & 8,000 & 2 \\
R23 & 6,80 & 7,40 & 7,80 & 392,50 & 3 & 8,000 & 3 \\
R17 & 5,80 & 7,00 & 6,80 & 276,08 & 6 & 7,000 & 4 \\
R22 & 6,80 & 7,00 & 5,80 & 276,08 & 7 & 7,000 & 5 \\
R21 & 4,80 & 8,40 & 6,60 & 266,11 & 8 & 7,000 & 6 \\
R9 & 2,20 & 9,20 & 8,60 & 174,06 & 11 & 7,000 & 7 \\
R19 & 6,00 & 8,80 & 6,40 & 337,92 & 4 & 6,000 & 8 \\
R14 & 5,80 & 9,00 & 6,00 & 313,20 & 5 & 6,000 & 9 \\
R13 & 5,40 & 8,80 & 4,40 & 209,09 & 9 & 6,000 & 10 \\
R16 & 5,40 & 7,20 & 5,20 & 202,18 & 10 & 6,000 & 11 \\
R3 & 2,00 & 8,80 & 7,20 & 126,72 & 13 & 6,000 & 12 \\
\hline
\end{tabular}




\begin{tabular}{llllllll}
\hline R7 & 6,60 & 4,80 & 4,00 & 126,72 & 14 & 6,000 & 13 \\
R1 & 2,40 & 7,20 & 7,00 & 120,96 & 15 & 6,000 & 14 \\
R5 & 4,80 & 5,80 & 6,00 & 167,04 & 12 & 5,000 & 15 \\
R6 & 4,60 & 6,20 & 4,20 & 119,78 & 16 & 5,000 & 16 \\
R11 & 4,80 & 6,60 & 3,20 & 101,38 & 17 & 5,000 & 17 \\
R18 & 5,40 & 7,00 & 2,40 & 90,72 & 18 & 5,000 & 18 \\
R12 & 3,20 & 6,40 & 3,60 & 73,73 & 19 & 4,000 & 19 \\
R15 & 4,80 & 6,00 & 2,20 & 63,36 & 20 & 4,000 & 20 \\
R10 & 3,00 & 5,60 & 3,20 & 53,76 & 21 & 3,000 & 21 \\
R4 & 2,00 & 6,40 & 2,20 & 28,16 & 22 & 3,000 & 22 \\
R20 & 1,40 & 4,40 & 2,20 & 13,55 & 23 & 3,000 & 23 \\
\hline
\end{tabular}

Table 5.6 Results of Fuzzy FMEA Integrated with FCM

\begin{tabular}{lllllllll}
\hline Risks & 0 & S & Re-S & D & $\begin{array}{l}\text { Fuzzy } \\
\text { RPN }\end{array}$ & $\begin{array}{l}\text { Old } \\
\text { Prioritization }\end{array}$ & Re-Fuzzy RPN & $\begin{array}{l}\text { New } \\
\text { Prioritization }\end{array}$ \\
\hline R8 & 8,2 & 8,6 & 10,00 & 9,4 & 9,000 & 1 & 9,000 & 1 \\
R2 & 6,8 & 9,2 & 10,00 & 8,2 & 8,000 & 2 & 8,000 & 2 \\
R23 & 6,8 & 7,4 & 9,047 & 7,8 & 8,000 & 3 & 8,000 & 3 \\
R22 & 6,8 & 7 & 10,00 & 5,8 & 7,000 & 5 & 7,000 & 4 \\
R17 & 5,8 & 7 & 9,065 & 6,8 & 7,000 & 4 & 7,000 & 5 \\
R21 & 4,8 & 8,4 & 10,00 & 6,6 & 7,000 & 6 & 7,000 & 6 \\
R7 & 6,6 & 4,8 & 7,190 & 4 & 6,000 & 13 & 7,000 & 7 \\
R9 & 2,2 & 9,2 & 9,557 & 8,6 & 7,000 & 7 & 7,000 & 8 \\
R19 & 6 & 8,8 & 10,00 & 6,4 & 6,000 & 8 & 6,000 & 9 \\
R14 & 5,8 & 9 & 10,00 & 6 & 6,000 & 9 & 6,000 & 10 \\
R16 & 5,4 & 7,2 & 10,00 & 5,2 & 6,000 & 11 & 6,000 & 11 \\
R5 & 4,8 & 5,8 & 7,611 & 6 & 5,000 & 15 & 6,000 & 12 \\
R13 & 5,4 & 8,8 & 8,800 & 4,4 & 6,000 & 10 & 6,000 & 13 \\
R1 & 2,4 & 7,2 & 10,00 & 7 & 6,000 & 14 & 6,000 & 14 \\
R6 & 4,6 & 6,2 & 8,471 & 4,2 & 5,000 & 16 & 6,000 & 15 \\
R3 & 2 & 8,8 & 10,00 & 7,2 & 6,000 & 12 & 6,000 & 16 \\
R11 & 4,8 & 6,6 & 9,085 & 3,2 & 5,000 & 17 & 6,000 & 17 \\
R18 & 5,4 & 7 & 10,00 & 2,4 & 5,000 & 18 & 6,000 & 18 \\
R12 & 3,2 & 6,4 & 8,667 & 3,6 & 4,000 & 19 & 6,000 & 19 \\
R15 & 4,8 & 6 & 7,062 & 2,2 & 4,000 & 20 & 5,000 & 20 \\
R4 & 2 & 6,4 & 9,051 & 2,2 & 3,000 & 22 & 5,000 & 21 \\
R10 & 3 & 5,6 & 8,324 & 3,2 & 3,000 & 21 & 4,000 & 22 \\
R20 & 1,4 & 4,4 & 5,031 & 2,2 & 3,000 & 23 & 3,000 & 23 \\
\hline
\end{tabular}

\section{Appendix A. Augmented Matrix}




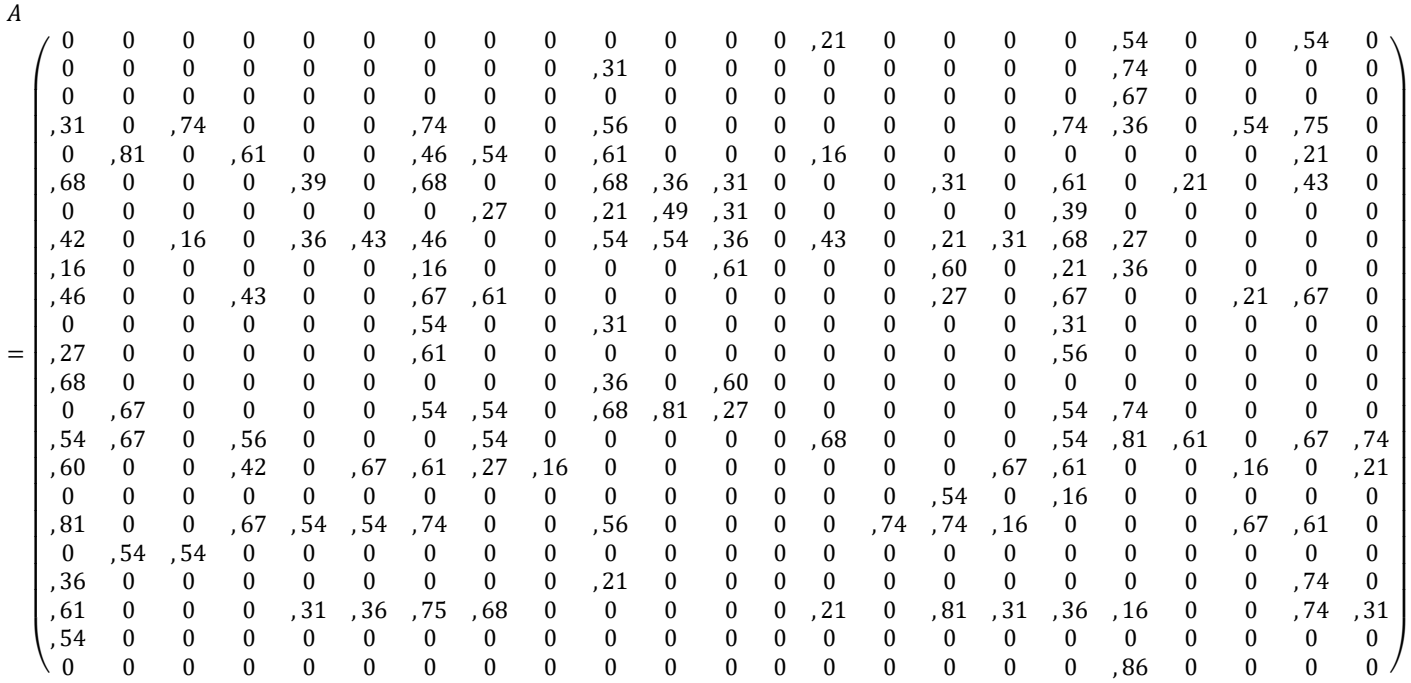

\section{Appendix B. FCM of IT Risks}

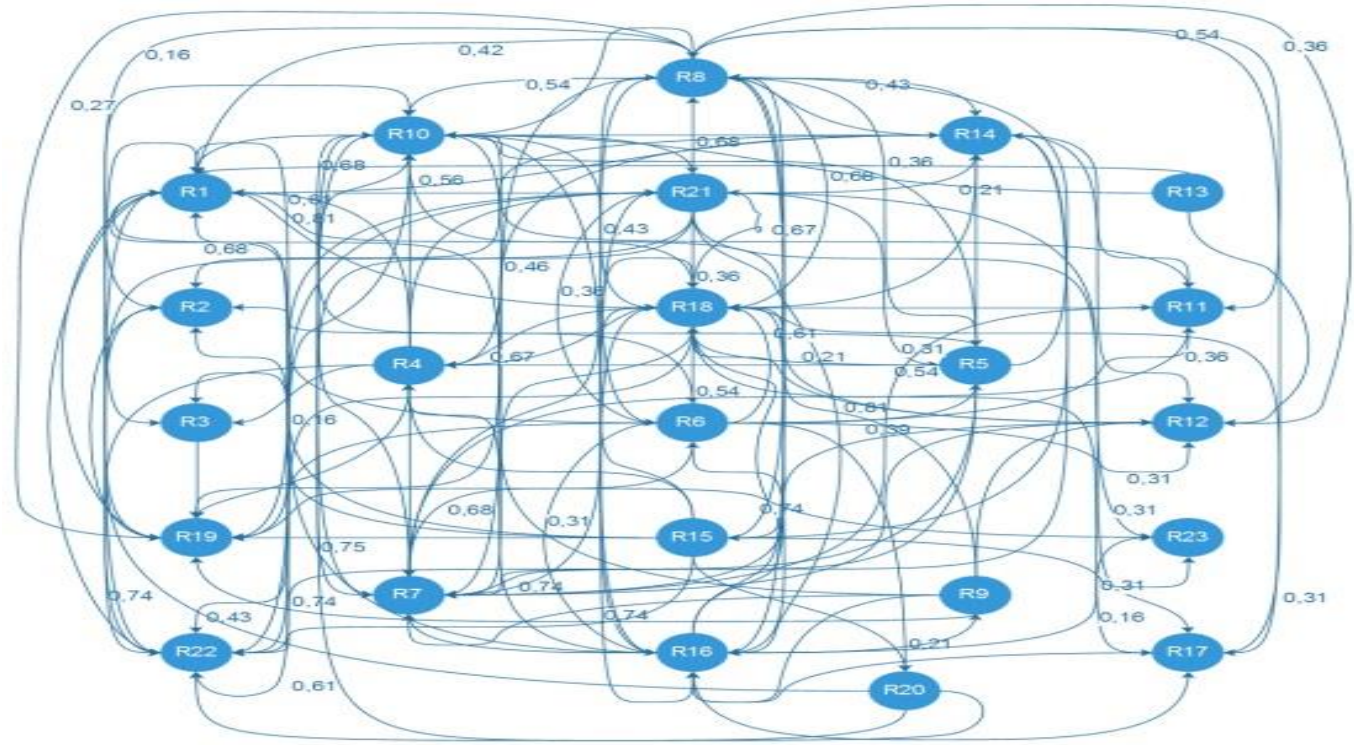

Appendix C. Initial Vector

$$
C_{1}=\left(\begin{array}{lllllllllllllllllllllll}
0 & 1 & 0 & 0 & 0 & 0 & 0 & 1 & 0 & 0 & 0 & 0 & 0 & 1 & 0 & 0 & 1 & 0 & 1 & 0 & 1 & 1 & 1
\end{array}\right)
$$

Appendix D. Steps of Simulation

\begin{tabular}{|c|c|c|c|c|c|c|c|c|}
\hline & C1 & C2 & C3 & C4 & C5 & C6 & C7 & C8 \\
\hline R1 & 0,000 & 0,827 & 0,986 & 0,995 & 0,995 & 0,995 & 0,995 & 0,995 \\
\hline R2 & 1,000 & 0,770 & 0,860 & 0,888 & 0,897 & 0,898 & 0,899 & 0,899 \\
\hline R3 & 0,000 & 0,668 & 0,732 & 0,789 & 0,795 & 0,796 & 0,796 & 0,796 \\
\hline
\end{tabular}




\begin{tabular}{lllllllll}
\hline R4 & 0,000 & 0,500 & 0,876 & 0,908 & 0,913 & 0,914 & 0,914 & 0,914 \\
R5 & 0,000 & 0,662 & 0,760 & 0,803 & 0,811 & 0,812 & 0,812 & 0,812 \\
R6 & 0,000 & 0,687 & 0,820 & 0,858 & 0,865 & 0,866 & 0,866 & 0,866 \\
R7 & 0,000 & 0,852 & 0,992 & 0,997 & 0,998 & 0,998 & 0,998 & 0,998 \\
R8 & 1,000 & 0,771 & 0,906 & 0,941 & 0,947 & 0,948 & 0,948 & 0,948 \\
R9 & 0,000 & 0,500 & 0,534 & 0,537 & 0,539 & 0,539 & 0,539 & 0,539 \\
R10 & 0,000 & 0,822 & 0,969 & 0,984 & 0,986 & 0,986 & 0,986 & 0,986 \\
R11 & 0,000 & 0,794 & 0,834 & 0,867 & 0,875 & 0,876 & 0,876 & 0,876 \\
R12 & 0,000 & 0,652 & 0,823 & 0,848 & 0,853 & 0,854 & 0,854 & 0,854 \\
R13 & 0,000 & 0,500 & 0,500 & 0,500 & 0,500 & 0,500 & 0,500 & 0,500 \\
R14 & 1,000 & 0,654 & 0,741 & 0,789 & 0,797 & 0,798 & 0,798 & 0,798 \\
R15 & 0,000 & 0,500 & 0,653 & 0,675 & 0,677 & 0,677 & 0,677 & 0,677 \\
R16 & 0,000 & 0,826 & 0,908 & 0,942 & 0,947 & 0,947 & 0,948 & 0,948 \\
R17 & 1,000 & 0,652 & 0,749 & 0,785 & 0,794 & 0,795 & 0,795 & 0,795 \\
R18 & 0,000 & 0,850 & 0,987 & 0,995 & 0,996 & 0,996 & 0,996 & 0,996 \\
R19 & 1,000 & 0,942 & 0,971 & 0,985 & 0,988 & 0,988 & 0,988 & 0,988 \\
R20 & 0,000 & 0,500 & 0,610 & 0,638 & 0,643 & 0,643 & 0,643 & 0,643 \\
R21 & 1,000 & 0,500 & 0,759 & 0,815 & 0,820 & 0,820 & 0,820 & 0,820 \\
R22 & 1,000 & 0,678 & 0,968 & 0,988 & 0,989 & 0,990 & 0,990 & 0,990 \\
R23 & 1,000 & 0,578 & 0,668 & 0,713 & 0,722 & 0,722 & 0,723 & 0,723 \\
\hline
\end{tabular}

\section{Appendix E. Graphical Notation of Simulation}
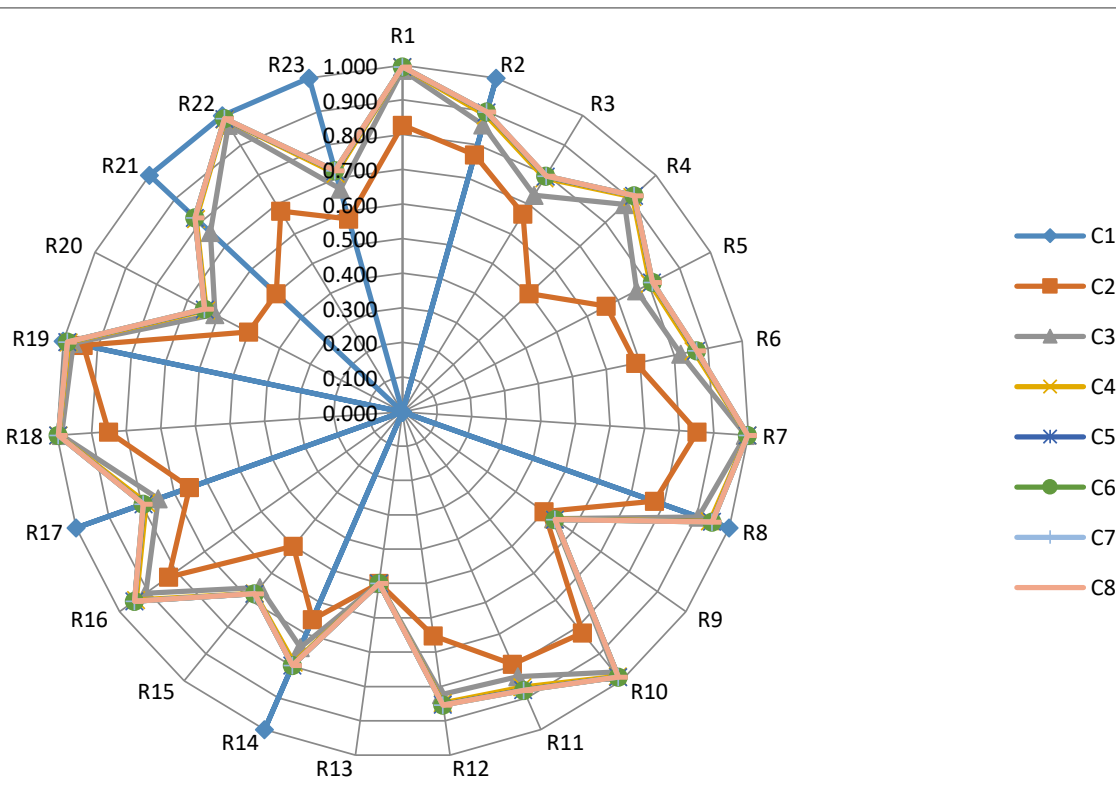

Appendix F. S Values and New S Values

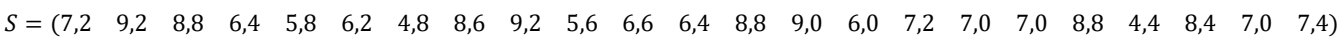
$S^{\prime}$

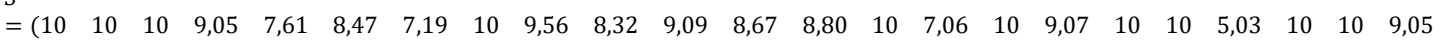




\section{References}

[1] Bowles, J. B. ve Pelaez, C. E. 1995, Fuzzy logic prioritization of failures in a system failure mode, effects and criticality analysis., Reliability Engineering and System Safety, Cilt 50, s. 203-213.

[2] Braglia, M. 2000, MAFMA: multi-attribute failure mode analysis. , International Journal of Quality \& Reliability Management, Cilt 17, s. 1017-1033.

[3] Bueno, S. ve Salmeron, J. L. 2009, Benchmarking main activation functions in fuzzy cognitive maps. , Expert Systems with Applications, Cilt 36, s. 5221-5229.

[4] Chang, K. H. 2009, Evaluate the orderings of risk for failure problems using a more general RPN methodology. , Microelectronics Reliability, Cilt 49, s. 1586-1596.

[5] Chuang, P. T. 2007, Combining service blueprint and FMEA for service design, Serv. Ind. J., Cilt 27, s. 91104.

[6] Grant, D. ve Osei-Bryson, K. 2005. Using fuzzy cognitive maps to assess MIS organizational change impact., 38th Annual Hawaii International Conference on System Sciences. s. 1-11.

[7] Kosko, B. 1986, Fuzzy cognitive maps. International Journal of Man-Machine Studies, Cilt 24, s. 65-75.

[8] Kosko, B. 1992, Neural networks and fuzzy systems. Englewood Cliffs, NJ: Prentice-Hall.

[9] Kumru, M. ve Kumru, P. Y. 2013, Fuzzy FMEA Application to Improve Purchasing Process in a Public Hospital, Applied Soft Computing, Cilt 13, s. 721-733.

[10] Lee, K. C., et al. 2002, Fuzzy cognitive map approach to web-mining inference amplification. Expert Systems with Applications, Cilt 22, s. 197-211.

[11] Lopez, Cristina ve Salmeron, Jose L. s.I. Dynamic risks modelling in ERP maintenance projects with FCM., Information Sciences, 2012.

[12] Narayanagounder, S. ve Gurusami, K. 2009, A new approach for prioritization of failure modes in design FMEA using ANOVA. , Proceedings of world academy of Sci. Eng. Techn.

[13] Papageorgiou, E. I. 2011, A new methodology for decisions in medical informatics using fuzzy cognitive maps based on fuzzy rule-extraction techniques., Applied Soft Computing, Cilt 11, s. 500-513.

[14] Pillay, A. ve Wang, J. 2003, Modified failure mode and effects analysis using approximate reasoning. , Reliability and System Safety, Cilt 79, s. 69-85.

[15] Puente, J., et al. 2002, A decision support system for applying failure mode and effects analysis. Int. J. Qual. Reliab. Manag., Cilt 19, s. 137-150.

[16] Rhee, S. J. ve Ishii, K. 2003, Using cost based FMEA to enhance reliability and serviceability, J. Adv. Eng. Inf., Cilt 17, s. 179-188.

[17] Stamatis, D. H. 2003, Failure Mode and Effect Analysis: FMEA from Theory to Execution, ASQC Quality Press.

[18] Tolman, E. C. 1948, Cognitive maps in rats and men. Psychological Review, Cilt 55, s. 189-208.

[19] Wang, Y. M., et al. 2009, Risk evaluation in failure mode and effects analysis using fuzzy weighted geometric mean. , Expert Systems with Applications, Cilt 36, s. 1195-1207.

[20] Xirogiannis, G. ve Glykas, M. 2007, Intelligent modeling of e-business maturity., Expert Systems with Applications, Cilt 32, s. 687-702.

[21] Yaman, D. ve Polat, S. 2009, A fuzzy cognitive map approach for effect-based operations: an illustrative case., Information Sciences, Cilt 179, s. 382-403.

[22] Zadeh, L. A. 3, 1965, Fuzzy sets. , Information and Control, Cilt 8, s. 338-353. 